\title{
Dynamical coupled-channel approaches on a momentum lattice
}

\author{
M. Döring ${ }^{1}$, J. Haidenbauer ${ }^{2,3}$, U.-G. Meißner ${ }^{1,2,3}$, and A. Rusetsky ${ }^{1}$ \\ 1 Helmholtz-Institut für Strahlen- und Kernphysik and Bethe Center for Theoretical Physics, Universität Bonn, \\ Nußallee 14-16, D-53115 Bonn, Germany \\ 2 Institute for Advanced Simulation and Jülich Center for Hadron Physics, Forschungszentrum Jülich, D-52425 Jülich, Germany \\ 3 Institut für Kernphysik, Forschungszentrum Jülich, D-52425 Jülich, Germany
}

the date of receipt and acceptance should be inserted later

\begin{abstract}
Dynamical coupled-channel approaches are a widely used tool in hadronic physics that allow to analyze different reactions and partial waves in a consistent way. In such approaches the basic interactions are derived within an effective Lagrangian framework and the resulting pseudo-potentials are then unitarized in a coupled-channel scattering equation. We propose a scheme that allows for a solution of the arising integral equation in discretized momentum space for periodic as well as anti-periodic boundary conditions. This permits to study finite size effects as they appear in lattice QCD simulations. The new formalism, at this stage with a restriction to $S$-waves, is applied to coupled-channel models for the $\sigma(600)$, $f_{0}(980)$, and $a_{0}(980)$ mesons, and also for the $\Lambda(1405)$ baryon. Lattice spectra are predicted.
\end{abstract}

PACS. 11.80.Gw Multichannel scattering - 12.38.Gc Lattice QCD calculations - 14.20.Gk Baryon resonances - 14.40.-n Hadrons, properties of mesons - 24.10.Eq Coupled-channel and distorted-wave models

\section{Introduction}

At low energies chiral perturbation theory has allowed for a comprehensive understanding of the strong interactions. At higher energies, where the chiral expansion starts to break down, rich spectra of excited states have been found in the meson-meson and meson-baryon sectors experimentally, see e.g. Ref. [1. Several analysis tools have been developed to disentangle the partial wave content and to pin down the resonance spectrum. Among those tools are dynamical coupled-channel models $2,3,4,5,5,6,7$, 8, 9, 10, characterized by interactions driven by hadron exchange, which are derived from effective Lagrangians. Such coupled-channel models usually fulfill two-body unitarity as well as some requirements of three-body unitarity and crossing symmetry. They are widely used for data analyses nowadays, for example by the Jülich 2, 3, 4, 5, 6, 7], EBAC [8] and DMT [10] groups in their investigations of the $\pi N$ system in the second and third resonance region.

In recent years information about the excited hadron spectrum is becoming available from the rapidly evolving field of lattice gauge theory. The masses of meson and baryon ground states could be determined at the few percent level [11] and close to physical pion masses. With regard to excited hadrons, signals of a rich spectrum could be found recently 12,13, 14, 15, 16 in calculations with pion masses $\geq 300 \mathrm{MeV}$ or in the quenched approximation. Of course, those excited states will be able to decay on the lattice, once the employed pion mass approaches the physical value. Then, resonance signals on the lattice manifest themselves in "avoided level crossing"; for narrow resonances, this signal is rather clear, but for broader resonances the avoided crossing becomes smeared out 17 . 18 .

In the one-channel- and also two-channel case, and given sufficient precision for the levels, the phase shift can be extracted unambiguously and without any model input by using Lüscher's formalism [19,20,21,22,23,24]. However, the precision of the lattice data is limited. Moreover, at higher energies often many more physical channels are open as it is the case, e.g., for the $\pi N$ interaction in the second and third resonance region. Thus, model input may be required to extract the physical properties and the resonance spectrum, in particular for broad resonances.

The physically realistic situation of multiple open decay channels is most conveniently organized in a coupledchannel scheme. Note that this is of particular importance in the study of finite size effects as discussed in detail, e.g., in Ref. 24]. Threshold openings show the same avoided level crossing as resonances, and it is, thus, necessary to take the physically allowed channels consistently into account, at least if they are known to couple strongly to the system of interest.

In Refs. 24, 25] a coupled-channel fit to synthetic lattice data with given errors has been carried out, stabilizing the extraction of phase shifts and pole positions by using Unitarized Chiral Perturbation Theory to expand the potential. The feasibility of this procedure has been shown 
and the error propagation from the lattice data to pole positions and phase shifts could be quantified.

In a similar way, from the point of view of hadron exchange approaches, the energy levels provided by lattice calculations can be treated as additional data that enter the combined analysis of hadronic reactions. Thus, the motivation of this study is to reformulate the formalism of the dynamical coupled-channel approaches in a manner that is suitable for a prediction of the discrete energy levels on a finite-size lattice. In particular, we find that the discretization of the multi-channel Lippman-Schwinger equation in the momentum space allows one to achieve the goal with a minimum effort. The paper contains a detailed discussion of such a discretization procedure.

The proposed framework can be used to produce the volume-dependent energy spectra for different physical systems. We are aware that present day lattice simulations do not provide more than a few data points for a limited number of volumes. Still, we would like to stress again that, as shown in Ref. [24, assuming a simple and sufficiently general parameterization for the chiral potentials, it is possible to extract the resonance parameters from a fit of the predicted energy levels to reasonably small data sets.

To demonstrate the discretization of dynamical coupledchannel approaches, in this study we utilize hadron-exchange potential models that have been developed to describe the meson-meson interaction in the strangeness $S=$ 0 sector [26, 27] and the meson-baryon interaction for $S=$ -1 as, e.g., in Refs. 28. 29. The meson-baryon sector with $S=0$ [2, 3, 4, 5, 6 is left to future studies.

In the aforementioned models, the interaction is given by $s^{-}, t$-, and $u$-channel exchange of hadrons, which connect all partial waves of the scattering amplitude. Furthermore, using $\mathrm{SU}(3)$ symmetry, one can also relate different reactions in the same framework, as recently demonstrated 6]. Thus, hadron exchange puts strong constraints on the coupled-channel amplitudes and provides at the same time a structured background from $t$ - and $u$-channel processes. Together with a minimal set of $s$-channel processes ("genuine" resonances) this allows for a reliable extraction of poles and residues from the analytic continuation of the amplitude [5].

The considered exchange processes constitute the interaction potential, $V$, which is then projected to partial waves that represent the basis in which the scattering equation is solved. Omitting channel indices, the scattering (Lippmann-Schwinger (LS)) equation in the center-ofmass frame reads [2, 3, 4, 5, 6, 28,

$$
\begin{aligned}
& T\left(q^{\prime \prime}, q^{\prime}\right)=V\left(q^{\prime \prime}, q^{\prime}\right) \\
& +\int_{0}^{\infty} d q q^{2} V\left(q^{\prime \prime}, q\right) \frac{1}{\sqrt{s}-E_{a}(q)-E_{b}(q)+i \epsilon} T\left(q, q^{\prime}\right),
\end{aligned}
$$

where $q^{\prime \prime} \equiv\left|\boldsymbol{q}^{\prime \prime}\right|\left(q^{\prime} \equiv\left|\boldsymbol{q}^{\prime}\right|\right)$ is the modulus of the outgoing (incoming) three-momentum that may be on- or offshell, $\sqrt{s}$ is the scattering energy, and $E_{a}=\sqrt{m_{a}^{2}+q^{2}}$ and $E_{b}=\sqrt{m_{b}^{2}+q^{2}}$ are the on-mass shell energies of the intermediate particles $a$ and $b$. Note that Eq. (1) is formulated in the partial wave basis, i.e. the amplitude only depends on the modulus of the incoming, outgoing, and intermediate particle momenta. The angular dependence of the full $T$-matrix is, for meson-baryon interaction, provided by the Wigner $d_{\lambda \lambda^{\prime}}^{J}(\vartheta)$-functions in the partial wave decomposition [28, where $\vartheta$ is the scattering angle and $\lambda\left(\lambda^{\prime}\right)$ is the helicity of the incoming (outgoing) baryon. For meson-meson interaction, the angular dependence reduces to the standard Legendre polynomials $P_{J}(\cos \vartheta)$. The solution $T$ of the LS equation allows to calculate the observables. Note that the pseudo-potential $V$ that appears on-shell and also half off-shell in Eq. (1) is fixed from the underlying interaction Lagrangian. In the following, we abbreviate the denominator in Eq. (11) as $\sqrt{s}-E_{\text {int }}$, i.e., $E_{\text {int }}=E_{a}(q)+E_{b}(q)$.

Dynamical coupled-channel models of the $\pi N$ interaction often take into account intermediate and final $\pi \pi N$ states $2,3,4,5,6,7,8,8$, though usually only in an effective way. Still, this complicates the structure of Eq. (11) 5]. Thus, for the present study we concentrate on physical problems where such three-body channels play only a minor role. The two interaction models we consider here [26, 28. do not contain any effective two-meson states. Also, note that the spherical symmetry on the lattice is broken, and partial waves mix; however, this effect is small for $S$ waves which mix only with waves of an orbital angular momentum $L \geq 4$ [20]. In this study, we concentrate on the formal development, while the issue of partial wave mixing and multi-meson intermediate states is left for further investigations.

\section{Discretization of the momentum space}

A standard way of solving the integral equation (11) for $T$ is to discretize the integral and invert a large matrix in the space of off-shell momenta. We will show in the following that with a few minor changes this scheme can be adapted to the discretized momentum space that corresponds to finite-size lattices as used in lattice gauge calculations. Explicitly, the integral term in the one-channel case is written in terms of the matrices $\mathbf{V}, \mathbf{G}$, and $\mathbf{T}$

$$
\begin{aligned}
& \int_{0}^{\infty} d q q^{2} \frac{V\left(q^{\prime \prime}, q\right) T\left(q, q^{\prime}\right)}{\sqrt{s}-E_{\mathrm{int}}} \rightarrow \mathbf{V} \mathbf{G} \mathbf{T} \\
& \mathbf{V}_{i j}=V\left(q_{i}, q_{j}\right), \quad \mathbf{T}_{i j}=T\left(q_{i}, q_{j}\right) \\
& \mathbf{G}=\operatorname{diag}\left(\frac{q_{1}^{2} w_{1}}{\sqrt{s}-E_{1}}, \cdots, \frac{q_{n}^{2} w_{n}}{\sqrt{s}-E_{n}}\right),
\end{aligned}
$$

where the $q_{i}$ 's are the sampling points $(i, j=1, \cdots, n)$ of the integration with the associated integration weights $w_{i}$, and $E_{i}$ is $E_{\text {int }}$ evaluated at $q_{i}$. The multi-channel case is discussed at the end of this section. The LS in its discretized form,

$$
\mathbf{T}=\mathbf{V}+\mathbf{V} \mathbf{G} \mathbf{T}
$$


reads component-wise for $i, j, k=1, \cdots, n$

$$
\mathbf{T}_{i j}=\mathbf{V}_{i j}+\sum_{k=1}^{n} \mathbf{V}_{i k} \frac{q_{k}^{2} w_{k}}{\sqrt{s}-E_{k}} \mathbf{T}_{k j}
$$

The solution $\mathbf{T}$ can now be obtained by matrix inversion, i.e.

$$
\mathbf{T}=(\mathbb{1}-\mathbf{V} \mathbf{G})^{-1} \mathbf{V}
$$

For the calculation of phase shifts or observables one needs the solution of the reaction amplitude $\mathbf{T}$ at the on-shell momenta. To obtain the on-shell result one can augment the matrices above by adding the on-shell momentum to the sampling points, i.e., $q_{n+1} \equiv q_{\text {on }}$, where $q_{\text {on }}$ fulfills $\sqrt{s}=\sqrt{m_{a}^{2}+q_{\mathrm{on}}^{2}}+\sqrt{m_{b}^{2}+q_{\mathrm{on}}^{2}}$. Then, $\mathbf{T}_{n+1, n+1}$ is the on-shell amplitude and $\mathbf{T}_{i, n+1}(i=1, \cdots, n)$ are the half off-shell amplitudes. Note that $\mathbf{G}_{n+1, n+1}=0$. Furthermore, the two-body singularity occurring at the on-shell energy in the LS equation (11) needs to be treated numerically. One possibility consists in rotating the sampling points into the complex plane 30. Another commonly used option is the Haftel-Tabakin scheme 31 in which the singular term in the evaluation of the integral in Eq. (2) is subtracted numerically and added analytically at the on-shell point.

For the discretization on the lattice, none of these two schemes needs to be employed, as we will see in the following. The lattice allows only for discrete momenta (periodic boundary conditions) which, for a simple cubic lattice, results in the substitution

$$
\begin{aligned}
\int \frac{d^{3} \boldsymbol{q}}{(2 \pi)^{3}} f(|\boldsymbol{q}|) & \rightarrow \frac{1}{L^{3}} \sum_{\boldsymbol{n}} f(|\boldsymbol{q}|), \\
\boldsymbol{q} & =\frac{2 \pi}{L} \boldsymbol{n}, \quad \boldsymbol{n} \in \mathbb{Z}^{3} .
\end{aligned}
$$

To apply the discretization to dynamical coupled-channel models, the LS equation (1) is rewritten as a three-dimensional integral and discretized according to Eq. (6). The three-dimensional summation can be further simplified by considering the multiplicities of the $i$-th neighbors for the simple cubic lattice with periodic boundary conditions, labeled as $(\mathrm{P})$ in the following. It can be shown that these multiplicities are given by the $\vartheta^{(\mathrm{P})}$-series $\left[\vartheta^{(\mathrm{P})}(i=0,1,2, \cdots)=1,6,12, \cdots\right]$, see Ref. 32]. The $\vartheta^{(\mathrm{P})}$ series is given by the coefficients of the Taylor expansion around $x=0$ of the function $g^{(\mathrm{P})}(x)=\left[\vartheta_{3}(0, x)\right]^{3}$,

$$
g^{(\mathrm{P})}(x)=\sum_{i=0}^{\infty} \vartheta^{(\mathrm{P})}(i) x^{i}
$$

where $\vartheta_{3}$ is the elliptic $\vartheta$ function [32],

$$
\vartheta_{3}(0, x)=\sum_{k=-\infty}^{\infty} x^{k^{2}}
$$

One obtains the scattering equation in discretized momentum space,

$$
\begin{aligned}
& T^{(\mathrm{P})}\left(q^{\prime \prime}, q^{\prime}\right)=V\left(q^{\prime \prime}, q^{\prime}\right) \\
& +\frac{2 \pi^{2}}{L^{3}} \sum_{i=0}^{\infty} \vartheta^{(\mathrm{P})}(i) \frac{V\left(q^{\prime \prime}, q_{i}\right) T^{(\mathrm{P})}\left(q_{i}, q^{\prime}\right)}{\sqrt{s}-E_{a}\left(q_{i}\right)-E_{b}\left(q_{i}\right)}, \quad q_{i}=\frac{2 \pi}{L} \sqrt{i} .
\end{aligned}
$$

Please note that the sampling points $q_{i}$ of the summation in Eq. (9) are different from those in Eq. (4) $\left(q_{i}\right.$ is the distance to the $i$-th neighbors). Note also that all quantities in this equation are real. Furthermore, $T^{(\mathrm{P})}$ will now have an infinite tower of poles, corresponding to the discrete spectrum of a system in a finite volume.

Finally, we can formulate convenient replacement rules to obtain Eq. (9) from existing dynamical coupled-channel models of the form of Eq. (3) as given in Refs. [2, 3, 4, 5, 6, 26, 27, 28, 29]. Comparing Eq. (4) with Eq. (9), we immediately obtain for the integration measure $q_{i}^{2} w_{i}$ and for the integration sampling points at $q_{i}$

$$
\begin{aligned}
q_{i}^{2} w_{i} & \rightarrow \frac{2 \pi^{2} \vartheta^{(\mathrm{P})}(i)}{L^{3}}, \\
q_{i} & =\frac{2 \pi}{L} \sqrt{i}, \quad i=0,1, \cdots,
\end{aligned}
$$

i.e. the first sampling point is at $q_{i}=0$ with $\vartheta^{(\mathrm{P})}(0)=$ 1. Note that if $V$ and $T$ do not depend on $q$, as it is the case, e.g., in the chiral unitary approaches considered in $33,34,35,36,37,38,39$, those quantities factorize from the integral in Eq. (2) so that the integral equation (11) reduces directly to an algebraic equation. The discretized amplitude can then can be rewritten, up to order $e^{-L}$ effects, in terms of the Lüscher zeta function $\mathcal{Z}_{00}$ within a $K$-matrix approach and for coupled channels as developed in Refs. 22, 23, 24. The influence of some $e^{-L}$ suppressed effects has been recently discussed in Ref. 24. Also in the present formalism, with a non-factorizing scattering equation, it would be possible to rewrite the discretized LS equation (9) in terms of $\mathcal{Z}_{00}$ (up to effects of order $\left.e^{-L}\right)$.

Hybrid boundary conditions [40,41,42 were introduced to distinguish scattering states from tightly bound quarkantiquark systems. Similarly, as proposed in Refs. 23. 24, twisted boundary conditions provide the possibility to change thresholds in lattice gauge calculations. This provides a unique opportunity to study the nature of resonances that lie close to a threshold [43, 44, 45, 46, 47] like, for example, the $f_{0}(980)$ with regard to the $\bar{K} K$ threshold, because the twisting moves the threshold while the resonance stays put. We realize that it could be quite challenging to implement this idea (including twisting for the sea quarks) in present-day lattice simulations.

Twisted boundary conditions, e.g. for the strange quark, lead to a change in the summation of the lattice momenta of the $\bar{K} K$ channel [23],

$$
\sum_{n} f(|\boldsymbol{q}|) \rightarrow \sum_{n} f(|\boldsymbol{q}+\boldsymbol{\theta} / L|)
$$


where $\boldsymbol{\theta}$ is the twisting angle that can be chosen to vary the $\bar{K} K$ threshold. For the present study of dynamical coupled-channel approaches, it is possible to formulate a scattering equation similar to Eq. (9), but with maximally twisted (i.e. anti-periodic) boundary conditions $\boldsymbol{\theta}=$ $(\pi, \pi, \pi)$. For this value of $\boldsymbol{\theta}$, the summation exhibits again a high symmetry: compared to the periodic case, the origin of the summation is simply shifted by $(1 / 2,1 / 2,1 / 2)$, i.e. it is in the center of the cubic unit cell. The multiplicity of the next neighbors is 8 , there are 24 nextto-next neighbors and so on. In general, the multiplicity of the $i$-th neighbors is given as 8 times the number of ordered ways of writing $n$ as the sum of 3 triangular numbers. We call this multiplicity $\vartheta^{(\mathrm{A})}$ in the following and $\vartheta^{(\mathrm{A})}(i=0,1,2, \cdots)=8,24,24, \cdots$. It can be shown that this series is given by the coefficients of the Taylor expansion [cf. Eq. (7)] around $x=0$ of the function $g^{(\mathrm{A})}(x)=\left[\vartheta_{2}(0, \sqrt{x})\right]^{3} x^{-3 / 8}$, where $\vartheta_{2}$ is the elliptic $\vartheta$ function 32 ,

$$
\vartheta_{2}(0, \sqrt{x})=2 x^{1 / 8} \sum_{k=0}^{\infty} x^{k(k+1) / 2} .
$$

With the new multiplicities $\vartheta^{(\mathrm{A})}$, and taking into account that the sampling points $q_{i}$ of the summation also change, the scattering equation with anti-periodic boundary conditions takes the form

$$
\begin{aligned}
T^{(\mathrm{A})}\left(q^{\prime \prime}, q^{\prime}\right) & =V\left(q^{\prime \prime}, q^{\prime}\right) \\
+ & \frac{2 \pi^{2}}{L^{3}} \sum_{i=0}^{\infty} \vartheta^{(\mathrm{A})}(i) \frac{V\left(q^{\prime \prime}, q_{i}\right) T^{(\mathrm{A})}\left(q_{i}, q^{\prime}\right)}{\sqrt{s}-E_{a}\left(q_{i}\right)-E_{b}\left(q_{i}\right)}
\end{aligned}
$$

with redistributed sampling points $q_{i}$,

$$
q_{i}=\frac{2 \pi}{L} \sqrt{\frac{8 i+3}{4}}, \quad i=0,1, \cdots .
$$

As before, $q_{i}$ is the distance to the $i$-th neighbors. Like for the periodic case, simple substitution rules with respect to the continuum formalism can be formulated. The integration weights change according to

$$
q_{i}^{2} w_{i} \rightarrow \frac{2 \pi^{2} \vartheta^{(\mathrm{A})}(i)}{L^{3}}
$$

and the distribution of the $q_{i}$ is given by Eq. (14) for the simple cubic lattice with anti-periodic boundary conditions.

So far, the discretization has been shown only for the one-channel case. The multi-channel case leads to no further complications and the formalism applies to it without changes. One just has to take into account that the quantities appearing in Eq. (4) depend also on the channel, for example $\mathbf{V}_{i j} \rightarrow \mathbf{V}_{i j}^{\mu \nu}$, where $\mu$ and $\nu$ characterize the outgoing and incoming channels. The sum runs now over both the momenta and the intermediate channels. The matrix G, cf. Eq. (2), is diagonal in channel space, too. If anti-periodic boundary conditions are imposed for, e.g., the strange quark in meson-meson scattering [23,24, the substitution rules of Eq. (15) are applied to the $\bar{K} K$ channel, while for the $\pi \pi$ channel the periodic rules of Eq. (10) are used. Note that in this case one has transition potentials $V^{\mu \nu}\left(q_{i}, q_{j}\right)$ with periodic $\left(q_{i}, \mu=\pi \pi\right)$ as well as anti-periodic $\left(q_{j}, \nu=\bar{K} K\right)$ sampling points together.

\section{Results and Conclusions}

In lattice calculations, the energy levels $E(L)$ can be obtained as a function of the box size $L$ of the simple cubic lattice, which allows to apply the Lüscher formalism [19. 20. to extract phase shifts. For the extraction of poles and phase shifts in the multi-channel case, see also Refs. 23. 24. Here, we simply predict the levels $E(L)$ applying the formalism developed in the previous section. The levels $E(L)$ are given by the singularities of the scattering equation (9) or (13), i.e. by the zeros of

$$
\operatorname{det}(\mathbb{1}-\mathbf{V} \mathbf{G})=0
$$

where $\mathbf{V}$ and $\mathbf{G}$ are now given by applying the substitution rules of Eq. (10) or Eqs. (14) and (15) to the quantities of Eq. (2), depending on whether periodic or anti-periodic boundary conditions are employed. Note that in a practical calculation the sums in Eqs. (9) and (13) need to be cut off at some $i_{\max }$ which is chosen in a way that the regularization schemes render contributions from $i>i_{\max }$ negligibly small (this can always be achieved). For the potential models studied in the present paper $i_{\max }$ had to be chosen in such a way 1 that $q_{i_{\max }} \approx 2.5-3 \mathrm{GeV}$.

We consider the two dynamical coupled-channel models mentioned in the Introduction. Specifically, we utilize the interaction potential developed in Refs. 26, 27] for the $I=0,1, S=0$ meson-meson sector for which the coupling between the $\pi \pi$ (or $\pi \eta$ ) and $\bar{K} K$ channels is taken into account. The $\sigma \equiv f_{0}(600), f_{0}(980)$, and $a_{0}(980)$ resonances all appear dynamically generated, i.e. without the need of corresponding $s$-channel exchanges. On the other hand, the model does include also a genuine resonance in form of an $s$-channel pole diagram, tentatively called $f_{0}(1400)$ in Ref. [26], whose bare mass is at $1520 \mathrm{MeV}$.

In the $I=0, S=-1$ meson-baryon sector, the dynamical coupled-channel model of Refs. [28,29] is considered. It includes the channels $\pi \Lambda, \pi \Sigma$, and $\bar{K} N$. Like in the meson-meson sector, the attraction in coupled channels

\footnotetext{
1 Note also that the inverse of $q_{i_{\max }}$ corresponds to distances of around $\simeq 0.07-0.08 \mathrm{fm}$. At these distances the form factors that provide the regularization render the high- $q$ contributions so small that the results practically do not change. Consequently, if the lattice spacing $a$ is taken less or equal to the above values (which are common values in present-day lattice simulations), the finite- $a$ artifacts are washed out and the limit $a \rightarrow 0$ can be safely performed, as was done in the present paper from the beginning. Still, a comprehensive treatment of systematic uncertainties tied to the finite spacing would require to address the actual lattice action that generates the levels, but that is beyond of what can be possibly done in the present framework.
} 


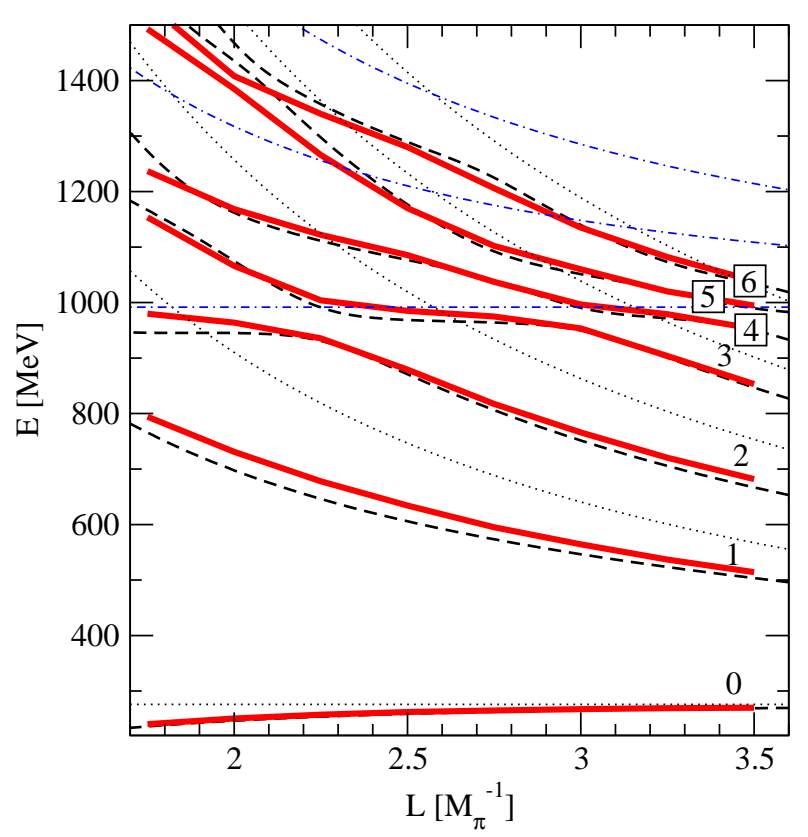

Fig. 1. Spectrum $E(L)$ for the $I=0, J^{P}=0^{+}$meson-meson sector $\left[\sigma(600), f_{0}(980)\right]$ for periodic boundary conditions. Solid lines: results for the dynamical coupled-channel approach of Refs. 26, 27]. Dashed lines: For comparison, the results for the chiral unitary approach of Ref. [33], see also Refs. [23, 24]. Dotted (dash-dotted) lines: free $\pi \pi(\bar{K} K)$ levels.

is so strong that the $\Lambda(1405)$ appears dynamically generated. In this approach, also the channels $\bar{K} \Delta, \bar{K}^{*} N$, and $\bar{K}^{*} \Delta$ are included. However, these channels contribute only effectively to the direct $\bar{K} N$ interaction in form of box diagrams [28]. Below the respective thresholds $(E<$ $1700 \mathrm{MeV}$ ) of these intermediate states, the discretized sum of Eq. (6) converges towards the integral in the large $L$ limit and, therefore, at this stage of the exploratory investigation we refrain from an explicit discretization of the integrals related to these box diagrams.

The calculated levels, using periodic boundary conditions, are shown by the solid lines in Figs. 1, 2, and 3, together with the respective free levels of the involved channels. In case of the meson-meson interaction the results exhibit a characteristic avoided level crossing at around $980 \mathrm{MeV}$ (more pronounced for $I=0$ than for $I=1$ ). In general, avoided level crossing is a signal for a resonance, but the avoided crossing is washed out for broader resonances. This applies for example to the broad $f_{0}(600)$ resonance as is obvious from Fig. 11. But it is also the case for the genuine $f_{0}(1400)$ resonance which acquires a significant width due to renormalization in the course of solving the Lippmann-Schwinger equation (11). Its pole is finally at $1346 \pm i 249 \mathrm{MeV}$, cf. Table V in Ref. [26], and thus it is almost as broad as the $f_{0}(600)$. In both cases no direct sign of the presence of these resonances is visible in the levels [cf. Fig. 1]. Nevertheless, in case of the $f_{0}(600)$ the pole position can be reconstructed from the levels (with large errors) by expanding a general pseudo-potential in powers of the scattering energy, as recently shown in Ref. [24. In

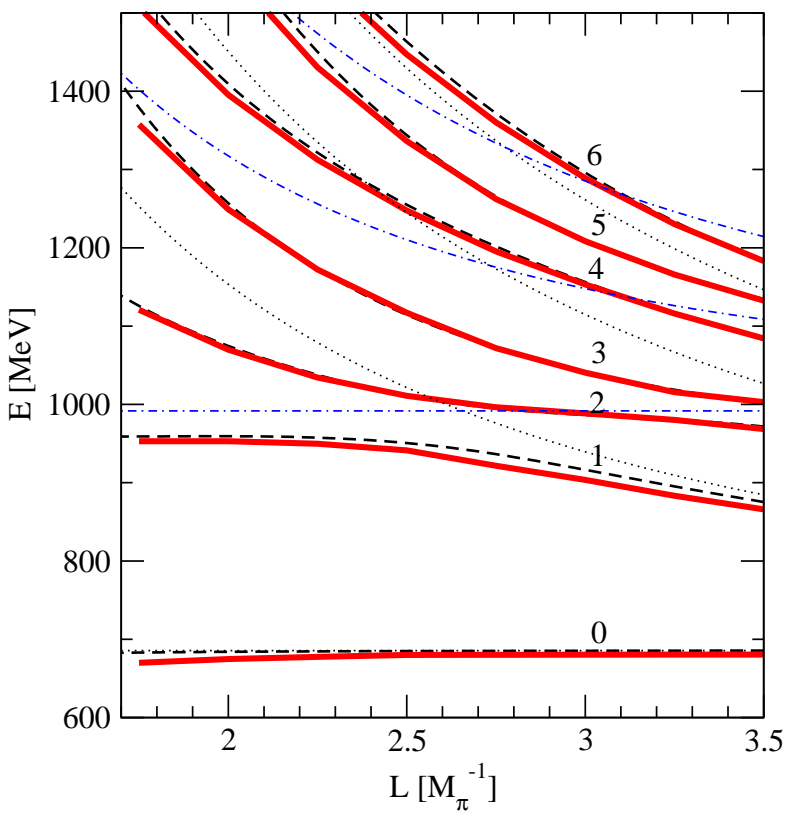

Fig. 2. Spectrum $E(L)$ for the $I=1, J^{P}=0^{+}$mesonmeson sector $\left[a_{0}(980)\right]$ for periodic boundary conditions. Solid lines: results for for the dynamical coupled-channel approach of Refs. 26 27]. Dashed lines: For comparison, the results for the chiral unitary approach of Ref. 33. Dotted (dash-dotted) lines: free $\pi \eta(\bar{K} K)$ levels.

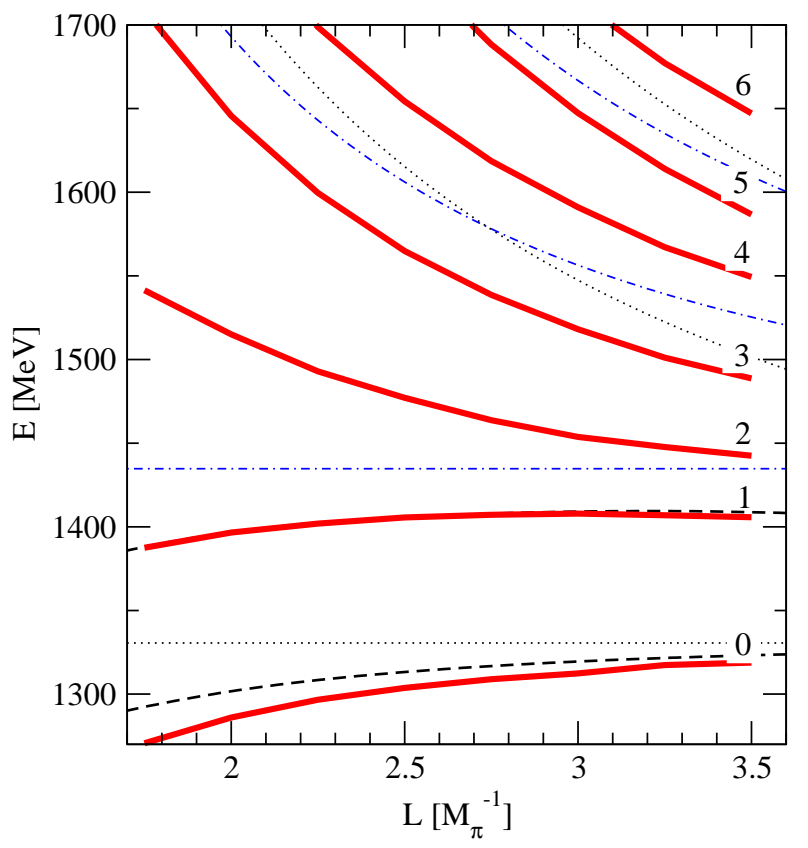

Fig. 3. Spectrum $E(L)$ of the $I=0, S=-1, J^{P}=\frac{1}{2}^{-}$mesonbaryon sector $[\Lambda(1405)]$ for periodic boundary conditions. Solid lines: results for the $\bar{K} N$ Jülich model of hadron exchange [28, 29. Dashed lines: For comparison, the first two levels as obtained from the chiral unitary approach of Ref. 37. Dotted (dash-dotted) lines: free $\pi \Sigma(\bar{K} N)$ levels. 
the future, we plan to address this (inverse) problem of how to reconstruct resonances from a given set of lattice data, in the present framework of hadron exchange.

Avoided level crossing is also present at particle thresholds. Resonances close to thresholds, like the $f_{0}(980)$ that is close to the $\bar{K} K$ threshold, require, thus, special attention. The avoided crossing, visible in Fig. 11 is therefore not a clear signal for the $f_{0}(980)$, but necessitates additional analysis. The inverse problem of reconstructing phase shifts and resonance poles from lattice levels, in such circumstances, has been addressed recently in Ref. [24], see also Ref. [48].

As mentioned above, the channel space in the $I=0$ sector comprises the $\pi \pi$ and the $\bar{K} K$ channels. The $4 \pi$ channel has been neglected as there are no phenomenological indications by the PDG [50] that require its inclusion at the energies of interest. Still, at energies beyond the $f_{0}$, the analysis could be refined by including these states, e.g. in terms of quasi-particle channels such as $\sigma \sigma$, $\rho \rho, \cdots$, as carried out, e.g. in Ref. [51]. In any case, the level structure of 4-body states is considerably more complicated than the one of two-body states considered here, and beyond the scope of this study.

In the meson-baryon sector, cf. Fig. 3, the level structure below the $\bar{K} N$ threshold is interesting because of a conjectured two-pole structure of the $1(1405)$ 38, 49 . Indeed, also the hadron-exchange approach of Ref. 28, considered here, predicts two poles below the $\bar{K} N$ threshold [29]. Note also the recent work of Ref. [52] in which, for the first time, the $\Lambda(1405)$ could be isolated in a lattice QCD calculation near the physical pion mass.

For the $I=S=0$ meson-meson case, we also apply the formalism for anti-periodic boundary conditions developed in the previous section. Corresponding result are presented in Fig. 4. The bending of the levels at the $\bar{K} K$ threshold from avoided level crossing disappears with anti-periodic boundary conditions as indeed clearly visible in the figure. This demonstrates again the usefulness of anti-periodic boundary conditions to study near-threshold resonances like the $f_{0}(980)$ discussed above [23,24.

For comparison, in Figs. 1, 2, and 4] we also show the spectra calculated in Refs. 23, 24, using the chiral unitary approach from Ref. 33 . As seen in the figures, the levels are similar to the result based on the hadron-exchange interaction but not identical, see Fig. 1. Indeed, also the respective phase shifts in the continuum case show noticeable differences [26, 33]. The further interpretation of the calculated spectra and observed small discrepancies between different approaches are left for future studies.

In conclusion, dynamical coupled-channel models can be modified so that the calculation of lattice spectra becomes possible, in agreement with the Lüscher formalism up to effects of order $e^{-L}$. This framework can be used to predict lattice spectra, as done here, or to analyze lattice spectra once lattice data will become available. Dynamical coupled channel approaches, which respect analyticity, unitarity and other general requirements of the $S$-matrix, can thus provide the opportunity to analyze and interpret

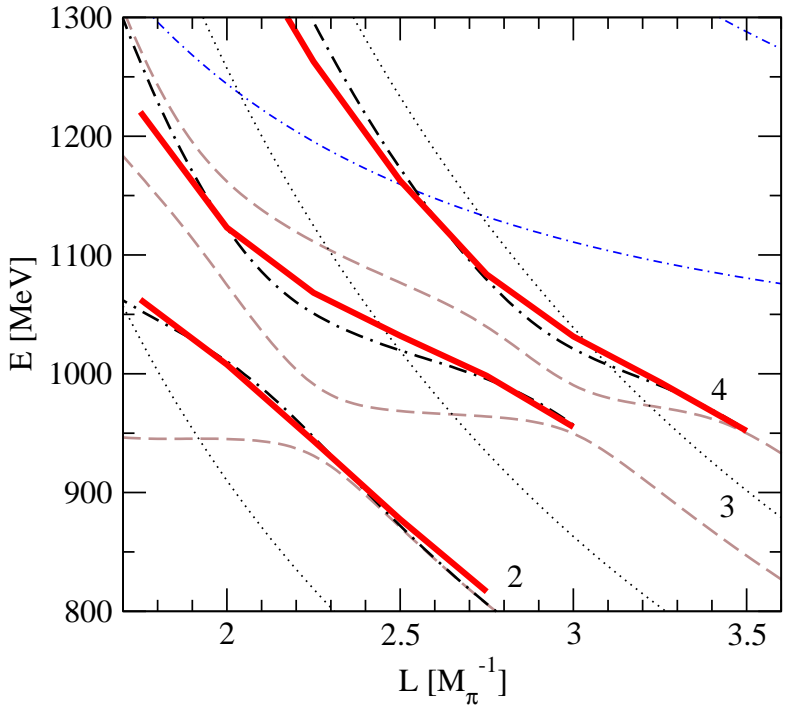

Fig. 4. Same as Fig. 1 (meson-meson, $I=0$ ) but with antiperiodic boundary conditions $(\theta=\pi)$ in the $\bar{K} K$ channel, for the dynamical coupled-channel model [26,27] (solid lines). For comparison, the result with anti-periodic boundary conditions for the chiral unitary approach of Ref. 33] is shown (thick dash-dotted lines). Also, the result with periodic boundary conditions of Fig. 1 is again shown (dashed lines). Dotted (thin dash-dotted) lines: free $\pi \pi$ (free anti-periodic $\bar{K} K$ ) levels.

both experimental data and lattice data within one single approach.

\section{Acknowledgments}

This work is supported by the Helmholtz Association by funds provided to the virtual institute "Spin and Strong QCD" (VH-VI-231), by the EU-Research Infrastructure Integrating Activity "Study of Strongly Interacting Matter" (HadronPhysics2, grant n. 227431) under the Seventh Framework Program of the EU, by the DFG (TR 16 and "Sachbeihilfe" GZ: DO 1302/1-2), and by COSY FFE under contract 41821485 (COSY 106). A.R. acknowledges support of the Georgia National Science Foundation (Grant \#GNSF/ST08/4-401).

\section{References}

1. E. Klempt and J. M. Richard, Rev. Mod. Phys. 82 (2010) 1095 arXiv:0901.2055 [hep-ph]].

2. O. Krehl, C. Hanhart, S. Krewald and J. Speth, Phys. Rev. C 62 (2000) 025207 arXiv:nucl-th/9911080.

3. A. M. Gasparyan, J. Haidenbauer, C. Hanhart and J. Speth, Phys. Rev. C 68 (2003) 045207 arXiv:nucl-th/0307072.

4. M. Döring, C. Hanhart, F. Huang, S. Krewald and U.-G. Meißner, Phys. Lett. B 681 (2009) 26 [arXiv: 0903.1781 [nucl-th]]. 
5. M. Döring, C. Hanhart, F. Huang, S. Krewald and U.-G. Meißner, Nucl. Phys. A 829 (2009) 170 [arXiv: 0903.4337 [nucl-th]].

6. M. Döring, C. Hanhart, F. Huang, S. Krewald, U.-G. Meißner and D. Rönchen, Nucl. Phys. A 851 (2011) 58 arXiv:1009.3781 [nucl-th]].

7. F. Huang, M. Döring, H. Haberzettl, J. Haidenbauer, C. Hanhart, S. Krewald, U.-G. Meißner and K. Nakayama, arXiv:1110.3833 [nucl-th].

8. B. Juliá Díaz, T. S. Lee, A. Matsuyama and T. Sato, Phys. Rev. C 76 (2007) 065201 arXiv:0704.1615 [nucl-th]].

9. M. W. Paris, Phys. Rev. C $\mathbf{7 9}$ (2009) 025208 arXiv:0802.3383 [nucl-th]].

10. L. Tiator, S. S. Kamalov, S. Ceci, G. Y. Chen, D. Drechsel, A. Švarc and S. N. Yang, Phys. Rev. C 82 (2010) 055203 arXiv:1007.2126 [nucl-th]].

11. S. Dürr et al., Science 322 (2008) 1224 arXiv:0906.3599 [hep-lat]].

12. C. McNeile and C. Michael [UKQCD Collaboration], Phys. Rev. D 74 (2006) 014508 arXiv:hep-lat/0604009.

13. N. Mathur, A. Alexandru, Y. Chen et al., Phys. Rev. D76 (2007) 114505 arXiv:hep-ph/0607110.

14. J. J. Dudek, R. G. Edwards, M. J. Peardon, D. G. Richards and C. E. Thomas, Phys. Rev. Lett. 103 (2009) 262001 arXiv:0909.0200 [hep-ph]].

15. J. Bulava et al., Phys. Rev. D 82 (2010) 014507 arXiv:1004.5072 [hep-lat]]

16. G. P. Engel, C. B. Lang, M. Limmer, D. Mohler and A. Schäfer [BGR Collaboration], Phys. Rev. D 82 (2010) 034505 arXiv:1005.1748 [hep-lat]].

17. V. Bernard, U.-G. Meißner and A. Rusetsky, Nucl. Phys. B 788 (2008) 1 arXiv:hep-lat/0702012.

18. V. Bernard, M. Lage, U.-G. Meißner and A. Rusetsky, JHEP 0808 (2008) 024 arXiv:0806.4495 [hep-lat]].

19. M. Lüscher, Commun. Math. Phys. 105 (1986) 153.

20. M. Lüscher, Nucl. Phys. B 354 (1991) 531.

21. X. Li et al. [CLQCD Collaboration], JHEP 0706 (2007) 053 arXiv:hep-lat/0703015.

22. M. Lage, U.-G. Meißner and A. Rusetsky, Phys. Lett. B 681 (2009) 439 arXiv:0905.0069 [hep-lat]].

23. V. Bernard, M. Lage, U.-G. Meißner and A. Rusetsky, JHEP 1101 (2011) 019 arXiv:1010.6018 [hep-lat]].

24. M. Döring, U.-G. Meißner, E. Oset and A. Rusetsky, Eur. Phys. J. A 47 (2011) 139 arXiv:1107.3988 [hep-lat]].

25. M. Döring and U.-G. Meißner, arXiv:1111.0616 [hep-lat].

26. G. Janssen, B. C. Pearce, K. Holinde and J. Speth, Phys. Rev. D 52 (1995) 2690 arXiv:nucl-th/9411021.

27. O. Krehl, R. Rapp and J. Speth, Phys. Lett. B 390 (1997) 23 arXiv:nucl-th/9609013.

28. A. Müller-Groeling, K. Holinde and J. Speth, Nucl. Phys. A 513 (1990) 557.

29. J. Haidenbauer, G. Krein, U.-G. Meißner and L. Tolos, Eur. Phys. J. A 47 (2011) 18 arXiv:1008.3794 [nucl-th]].

30. R. Aaron and R. D. Amado, Phys. Rev. 150 (1966) 857.

31. M. Haftel, F. Tabakin, Nucl. Phys. A 158 (1970) 1.

32. For tabulated numbers and further references see, e.g., The On-Line Encyclopedia of Integer Sequences, http://oeis.org/A005875 for the $\vartheta^{(\mathrm{P})}$ series and http://oeis.org/A008443 for the $\vartheta^{(\mathrm{A})}$ series.

33. J. A. Oller and E. Oset, Nucl. Phys. A 620 (1997) 438; [Erratum-ibid. A 652 (1999) 407] arXiv:hep-ph/9702314.

34. N. Kaiser, Eur. Phys. J. A3 (1998) 307.
35. J. A. Oller, E. Oset and J. R. Pelaez, Phys. Rev. D 59 (1999) 074001; $\quad$ [Erratum-ibid. D $\quad 60$ (1999) 099906]; [Erratum-ibid. D 75 (2007) 099903] arXiv:hep-ph/9804209.

36. U.-G. Meißner and J. A. Oller, Nucl. Phys. A 673 (2000) 311. arXiv:nucl-th/9912026.

37. E. Oset and A. Ramos, Nucl. Phys. A 635 (1998) 99 arXiv:nucl-th/9711022.

38. D. Jido, J. A. Oller, E. Oset, A. Ramos, U.-G. Meißner, Nucl. Phys. A725 (2003) 181 nucl-th/0303062.

39. C. Garcia-Recio, M. F. M. Lutz and J. Nieves, Phys. Lett. B 582 (2004) 49 arXiv:nucl-th/0305100.

40. F. Okiharu et al., arXiv:hep-ph/0507187.

41. H. Suganuma, K. Tsumura, N. Ishii and F. Okiharu, PoS LAT2005 (2006) 070 arXiv:hep-lat/0509121.

42. H. Suganuma, K. Tsumura, N. Ishii and F. Okiharu, Prog. Theor. Phys. Suppl. 168 (2007) 168 arXiv:0707.3309 [heplat]].

43. V. Baru, J. Haidenbauer, C. Hanhart, Yu. Kalashnikova and A. E. Kudryavtsev, Phys. Lett. B 586 (2004) 53 arXiv:hep-ph/0308129.

44. V. Baru, J. Haidenbauer, C. Hanhart, A. E. Kudryavtsev and U.-G. Meißner, Eur. Phys. J. A 23 (2005) 523 arXiv:nucl-th/0410099.

45. M. Döring, Nucl. Phys. A $\mathbf{7 8 6}$ (2007) 164 arXiv:nucl-th/0701070.

46. M. Döring and K. Nakayama, Eur. Phys. J. A 43 (2010) 83 arXiv:0906.2949 [nucl-th]].

47. P. C. Bruns, M. Mai and U.-G. Meißner, Phys. Lett. B 697 (2011) 254 arXiv:1012.2233 [nucl-th]].

48. A. M. Torres, L. R. Dai, C. Koren, D. Jido, E. Oset, arXiv:1109.0396 [hep-lat]].

49. J. A. Oller and U.-G. Meißner, Phys. Lett. B 500 (2001) 263 arXiv:hep-ph/0011146.

50. K. Nakamura et al. [ Particle Data Group Collaboration ], J. Phys. G G37 (2010) 075021.

51. M. Albaladejo, J. A. Oller, C. Piqueras, Int. J. Mod. Phys. A24 (2009) 581-585. arXiv:0804.2341 [hep-ph]].

52. B. J. Menadue, W. Kamleh, D. B. Leinweber, M. S. Mahbub, arXiv:1109.6716 [hep-lat]]. 